Ann. Biol. anim. Bioch. Biophys., I966, 6 (3), 427-429.

\title{
INTERACTION ENTRE DEUX LOCI POUR UNE SÉGRÉGATION ANORMALE GHEZ LA POULE
}

\author{
P. MÉRAT \\ Station de Recherches avicoles, \\ Centre national de Recherches zootechniques, 78 -Jouy-en-Josas
}

Le locus $R$, chez la poule, détermine la forme de la crête, et comporte deux allèles : $\mathrm{R}$ (crête "en rose "), dominant, et $r$ (crête " simple "), récessif. La dominance de $\mathrm{R}$ a toujours été trouvée complète. Il en est de même pour la pénétrance de l'allèle $r$ à l'état homozygote.

Nous avons déjà fait mention (MÉRAT, I962) d'une ségrégation anormale se traduisant, dans la descendance du croisement $\mathrm{R} r \times r r$, par un excès de mâles à crête simple et par un défaut de mâles à crête en rose vis-à-vis du nombre de femelles de la même catégorie. Les nombres observés de mâles à crête en rose, de mâles à crête simple, de femelles à crête en rose, de femelles à crête simple, à l'éclosion, étaient respectivement les suivants : I I79, I 39I, I 212, I 2 I 9.

Nos données suggéraient (Mérat, rg63) que l'anomalie en cause ne devait pas être postérieure à la formation des zygotes, et ne pouvait s'expliquer par une dominance variable de l'allèle $\mathrm{R}$. En effet, quant au premier point, le taux d'éclosion dans les familles présentant la proportion la plus perturbée est élevé dans l'ensemble, et il n'est pas inférieur à celui des autres familles. D'autre part, l'examen de la proportion des sexes pour chaque type de crête montre qu'il existe, non seulement un défaut de mâles à crête en rose, mais aussi un excès de mâles à crête simple. Pour le deuxième point, un test de descendance de coqs à crête simple issus de familles à ségrégation perturbée n'en a jamais révélé aucun qui fût du génotype $\mathrm{R} r$.

Une information supplémentaire provient de ce que certaines familles étudiées comportaient aussi une disjonction mendélienne au locus $\mathrm{C}$ de coloration du plumage ( $\mathrm{C}=$ plumage coloré, $c=$ absence de pigmentation), donnant le croisement de dihybridisme :

ou

$$
\begin{aligned}
& \text { o } C c \mathrm{R} r \times \text { 車 } c c r r \\
& o \mathrm{C} c \mathrm{R} r \times q \mathrm{C} c r r
\end{aligned}
$$

Nous avons totalisé pour ces deux croisements, dans chaque sexe, les nombres observés pour les quatre catégories de zygotes " colorés à crête en rose ", " colorés à 
crête simple ", "blancs à crête en rose ", "blancs à crête simple ", symbolisées respectivement $(\mathrm{CR}),(\mathrm{C} r)$, et ainsi de suite. Le résultat est indiqué dans le tableau I. On voit que la proportion des types de crête n'est anormale que chez les mâles colorés; il y a, parmi eux, excès de crêtes simples.

TABLEAU I

Nombres observés sur l'ensemble des familles

\begin{tabular}{|c|c|c|c|c|c|c|c|c|}
\hline \multirow{2}{*}{$\begin{array}{l}\text { Type de croisement } \\
\text { pour } \mathrm{C} \text { et } c\end{array}$} & \multicolumn{4}{|c|}{ Nombre de mâles } & \multicolumn{4}{|c|}{ Nombre de femelles } \\
\hline & (CR) & $(\mathrm{Cr})$ & $(c \mathrm{R})$ & $(c r)$ & $(\overline{\mathrm{CR}})$ & $(\mathrm{C} r)$ & $(c \mathrm{R})$ & $\overline{(c r)}$ \\
\hline $\mathrm{C} c \times c c$ & 215 & 268 & 246 & 216 & 211 & 215 & 220 & 212 \\
\hline $\mathrm{C} c \times \mathrm{C}_{c} \ldots$ & 110 & 167 & 36 & 50 & 152 & 133 & 40 & 48 \\
\hline
\end{tabular}

En nous limitant, d'autre part, aux familles où la proportion des types de crête chez les mâles est différente de I/I au seuil $5 \mathrm{p}$. Ioo, nous obtenons le tableau suivant (où le total des deux croisements est utilisable pour comparer la proportion des types de crête parmi les individus colorés et parmi les blancs) :

\section{TABLEAU 2}

Familles à proportion la plus perturbée pour les crêtes: effectifs des quatre types de zygotes, par sexe

\begin{tabular}{|c|c|c|c|c|c|c|c|c|}
\hline \multirow{2}{*}{$\begin{array}{l}\text { Type de croisement } \\
\text { pour C et } c\end{array}$} & \multicolumn{4}{|c|}{ Mâles } & \multicolumn{4}{|c|}{ Femelles } \\
\hline & (CR) & $(\mathrm{C} r)$ & $(c \mathrm{R})$ & (cr) & (CR) & $(\mathrm{C} r)$ & $(c \mathrm{R})$ & $(c r)$ \\
\hline $\begin{array}{l}\mathrm{C} c \times c c . \\
\mathrm{C} c \times \mathrm{C} c_{c} .\end{array}$ & $\begin{array}{r}53 \\
5\end{array}$ & $\begin{array}{r}105 \\
21\end{array}$ & $\begin{array}{r}76 \\
4\end{array}$ & $\begin{array}{r}79 \\
1\end{array}$ & $\begin{array}{l}65 \\
10\end{array}$ & $\begin{array}{l}75 \\
10\end{array}$ & $\begin{array}{r}67 \\
2\end{array}$ & $\begin{array}{r}64 \\
4\end{array}$ \\
\hline Total .... & 58 & 126 & 80 & 80 & 75 & 85 & 69 & 68 \\
\hline
\end{tabular}

Ici encore, c'est seulement chez les mâles colorés que la proportion des types de crête apparaît anormale : le test de conformité de cette proportion vis-à-vis de la proportion I/I donne un $\chi^{2}$ égal à $25, \mathrm{I} 30$ pour un degré de liberté $\left(\mathrm{P}<\mathrm{IO}^{-6}\right)$. Quant au $\chi^{2}$ de contingence entre mâles colorés et blancs pour la répartition des crêtes, il est égal à $\mathrm{x} 2, \mathrm{I} 5^{8}(\mathrm{P}<0, \mathrm{OOI})$.

La proportion globale des mâles colorés et des blancs dans chacun des deux croisements est visiblement normale.

Chez les femelles, toutes les proportions sont conformes aux prévisions.

Rappelons que les loci $\mathrm{C}$ et $\mathrm{R}$ ne présentent entre eux aucun linkage et correspondent à des gènes à pénétrance complète, et à des phénotypes observables dès l'éclosion. 
Le résultat présent renforce nos conclusions précédentes (MÉRAT, I963) quant à 1a difficulté d'expliquer les ségrégations anormales constatées par une mortalité zygotique, quel qu'en soit le stade.

Un problème supplémentaire est posé du fait de la restriction de l'anomalie à une fraction seulement des gamètes mâles fécondants, et de l'interaction entre deux loci pour le phénomène en question, outre le rôle joué par la présence ou l'absence du chromosome lié au sexe chez l'ovule.

$$
\text { Reçu pour publication en mai } 1966 .
$$

\title{
SUMMARY
}

\author{
INTERACTION BETWEEN TWO LOCI FOR AN ABNORMAL SEGREGATION IN THE FOWL
}

In crosses of the types $\delta \mathrm{R} r \mathrm{C} c \times$ o $r r c c$ or $\delta \mathrm{R} r \mathrm{C} c \times$ o $r r \mathrm{C} c$, we observed an excess of single comb male progeny and a shortage of rose-comb males, compared with the expected numbers and the numbers of females in the corresponding classes. This anomaly was restricted to the coloured birds ( $\mathrm{C} c$ or $\mathrm{CC}$ ). The proportion of comb types was normal among white $(c c)$ birds. This result confirms our previous interpretation of abnormal segregations to the $\mathbf{R}$ locus by a phenomenon of selective fertilization.

\section{RÉFÉRENCES BIBLIOGRAPHIQUES}

Mérat P., I962. Ségrégations anormales pour les allèles "crête simple " et "crête en rose " chez la poule. I. Croisement of $\mathrm{Rr} \times \% \mathrm{rr}$. Ann. Biol. anim. Bioch. Biophys., 2, Iog-1 17 .

Mérat P., I963. Ségrégations anormales pour les allèles "crête simple " et "crête en rose " chez la poule.

IV. Discussion d'ensemble relative aux trois types de croisement. Ann. Biol. anim. Bioch. Biophys., 3, I 33-I 4 I. 